\title{
Estimating Wind Turbines Mechanical Constants
}

\author{
A.G. González Rodríguez ${ }^{1}$, A. González Rodríguez ${ }^{2}$ and M. Burgos Payán ${ }^{3}$ \\ ${ }^{1}$ Department of Electronic, University of Jaén \\ Escuela Politécnica Superior - Campus "Las Lagunillas" - 23071 Jaén, Spain \\ phone: +34 953212424 - fax: +34 953212400 - e-mail: agaspar@ujaen.es \\ ${ }^{2}$ Department of Applied Mechanical and Project Engineering, University of Castilla la Mancha \\ Avda. Camilo José Cela s/n - 13071 Ciudad Real, Spain \\ phone: +34 926295300 - fax: +34926 295361- e-mail: Antonio.Gonzalez@uclm.es \\ ${ }^{3}$ Department of Electrical Engineering, University of Seville \\ Camino de los Descubrimientos, $\mathrm{s} / \mathrm{n}-41092$ Sevilla, Spain \\ phone: +34 954487283 - fax: +34 954487284 - e-mail: mburgos@us.es
}

\begin{abstract}
The performance of wind turbines during transient situations such as the soft-starting process or in transient stability studies is strongly influenced by a broad set of mechanical parameters involved in the turbine dynamics, mainly the inertia constant, self and mutual damping and torsional stiffness. The values of these parameters use to be difficult to find because the wind turbine manufacturer/supplier usually do not supply this information. So researchers are usually forced to use vague estimated values to simulate these transients.

This communication presents an analysis of the mechanical component that models the wind turbine rotor, as well as its mechanical coupling to the generator shaft. A discussion will be introduced to question how to calculate approximate but realistic values for the wind turbine inertia time constant, preferably as a function of the blade mass and length, but also the rated wind turbine power. The dependence of the remaining mechanical wind turbine parameter values will be also provided.
\end{abstract}

\section{Key words}

Wind turbine rotor, inertia time constant, estimation of mechanical parameters.

\section{Introduction}

The inertia constant has a great and direct impact on the transient stability of wind farms and the start-up of wind turbines [1], and thereby this value is referenced continuously in wind farm dynamic performance studies. However, most of these papers only offer an estimation of inertia as suppliers do not use to facilitate this information. Thus, disregarding papers about vertical axe wind turbines, two-bladed rotors or small size machines, very few works indicate numerical values for this parameter [2-6], most of them being estimated values, as in [6], where Akhmatov obtains the inertia values for the rotor and generator from an experiment where turbine is abruptly disconnected from the grid, or refer to multi-megawatt wind turbines [7-8]. With regard to simulations it is common to find the same experiment for several inertia values [6, 9-11].
The aim of this study is to provide an expression to estimate the inertia time constant for a typical blade if no reliable data are available. Realistic values for the rest of mechanical parameters will be also presented.

Section 2 will present an overview of the mechanical parameters to be considered in a transient simulation of a wind turbine.

In Section 3, the shape and components of a typical blade will be shown.

An approximate static analysis will be reported in Section 4 to determine the theoretical distribution of the thickness of the skin and reinforcement box that must withstand the momenta produced by the aerodynamical forces. A more practical distribution will be also analyzed and compared to typical ones extracted from the bibliography. From both kinds of distributions it can be deduced that there is a relationship linking the inertia constant with the blade weight and the square of the blade length.

In Section 5, available technical data and the previous relationship for the inertia constant will allow us to obtain expressions for the inertia time constant as a function of the turbine capacity and the rotor diameter.

With regard to the remaining mechanical parameters, values will be provided extracted from the bibliography and catalogues, as well as useful expressions and dependences.

Section 8 will present the conclusions of this study.

\section{Wind turbine mechanical parameters. An overview}

A three-bladed horizontal axis rotor turbine has been considered is this paper, as it is the most common 
configuration. Figure 1 shows, in the upper side, one of the possible mechanical configurations. As can be seen there is a flexible coupling to allow for possible small misalignments between the turbine shaft and the generator and to absorb torque variations, reducing in this way the material fatigue, which is an important issue in this kind of equipment. In this configuration, there is a mechanical brake on the fast shaft, where the braking torque is lower, but with the drawback of idleness in case of gearbox or coupling breakage.

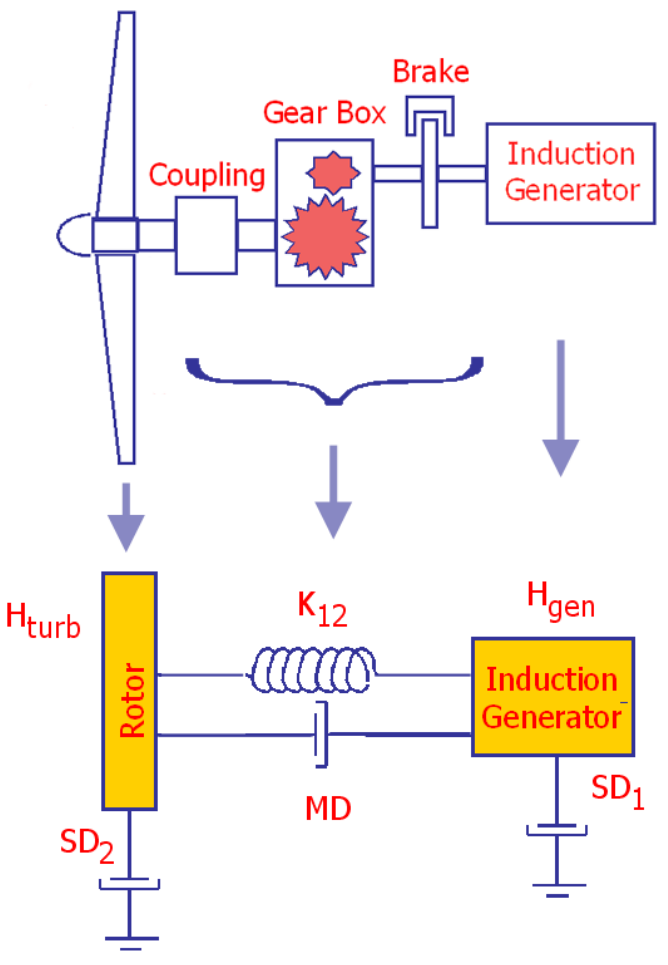

Fig. 1. Graphical model for a multi-mass mechanical dynamics.

In the lower side, the parameters of the dynamical model are presented. The wind turbine can be modelled by two masses connected to a single rotating shaft. The first mass represents the induction generator. The second one refers to the wind turbine.

The most significant components in the wind turbine dynamics are the induction generator inertia constant, $H_{G E N}$, and, above all, the turbine inertia, $H_{T U R B}$, due to the blade length and weight.

Another important parameter is the self damping of every mass. For the turbine, $S D_{2}$, this parameter accounts for the aerodynamical drag producing a torque which opposes the wind motor torque. It depends on the blade length, chord, cleanliness and material, as well as the relative wind velocity in relation to the blade velocity, specially regarding to the speed regime (turbulent or laminar). Torque produced by the aerodynamical drag is proportional to the square of the speed. With regard to the generator self damping, $S D_{l}$, it is due to the friction of the rotor shaft and to the ventilation losses.
In a simplified way, torque transmission is enabled by the torsion in the fast and slow shafts. In any case a flexible coupling is usually introduced as previously mentioned. In this case, torque transmission takes place due to the coupling twist angle that is inversely proportional to the shaft spring constant or torsional stiffness, $K_{12}$, and directly proportional to the transmitted torque.

When transients in the transmitted torque occur, variations will appear in the twist angle. These variations are damped by a torque proportional to the difference of speeds between both rotating masses. The proportionality coefficient is the mutual damping parameter, $M D$. Therefore, shaft spring constant and mutual damping values are determined by the coupling between turbine rotor and the gearbox (as in Fig. 1), or between gearbox and generator.

Summarizing, the parameters to be estimated or, at least, bounded are those shown in Fig. 1: turbine and generator inertia time constants $H$, turbine and generator self damping $S D$, torsional stiffness $K$ of the flexible coupling between rotor and generator, and mutual damping $M D$ of the changes in the twist angle of this coupling.

\section{Geometry and components of a blade}

The inertia constant has a great and direct impact on the wind farms transients, and thereby this value is referenced continuously in wind farm dynamic performance studies.

An estimation of the inertia constant can be obtained starting from the blade geometry and cross-section, and calculating an approximated mass distribution along the blade span. One possible inner structure [12] with some necessary definitions appears in Fig. 2.

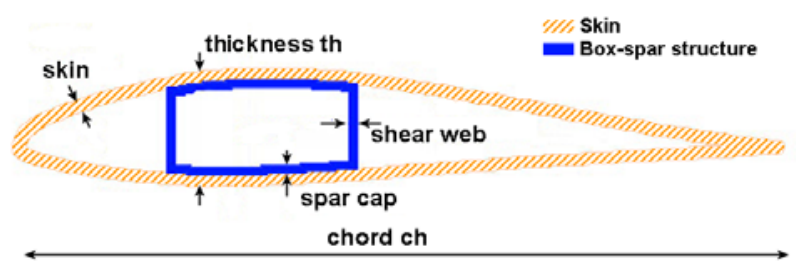

Fig. 2. Cross section of a wind turbine blade.

From the point of view of the structural resistance of the blade, it is made of a glass reinforced epoxy, disposed in an outer skin and a box-spar structure acting as the structural reinforcement for the blade to be more efficient at resisting out-of-plane shear loads and bending momenta.

Defining the thickness as the maximum length in the direction transversal to the chord line, and relative thickness as this magnitude divided by the chord, the span-wise distribution of this value offers the approximated distribution of Fig. 3. The distribution of the chord is also shown. 


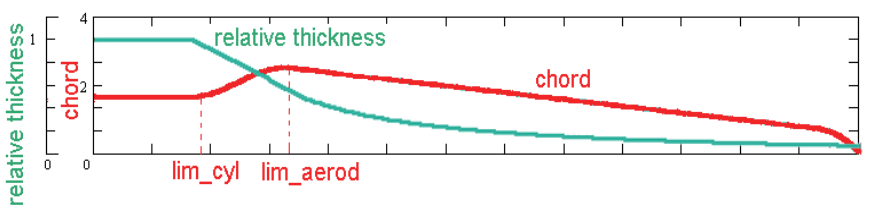

Fig. 3. Chord and relative thickness distribution along the span.

Next to the junction with the hub there is a cylindrical constant-chord part. The longer and more important part is the farther from the root and it has an aerodynamical shape as in Fig. 2, more elongated towards the tip and wider towards the root. Between both parts there is a complicated variable geometry link that fits both kinds of sections.

Fig. 4 shows a typical blade obtained from the previous profile and distributions.

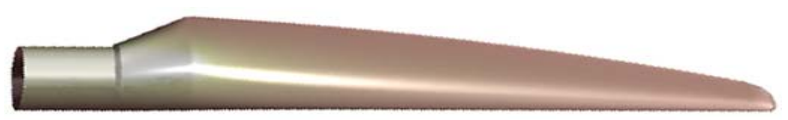

Fig. 4. A typical wind turbine blade.

Starting from the blade geometry, an estimation of the mass distribution, which in turn determines the inertia time constant, will be obtained. If the width of the skin, the shear webs and the spar caps are considered as constants, the mass distribution, and hence the inertia time constant, can be easily obtained. It would only be necessary to know the mass and length of the blade, and the hub diameter. The following section will provide an estimative evolution for these widths obtained from the study of the loads acting on the blade.

\section{Static strength analysis}

\section{A. Approximations and fatigue study}

In order to provide a qualitative idea of the mass distribution along the blade, some approximations will be done:

- It will be assumed that the mass is due to the glass reinforced epoxy material of the skin and the boxspar structure (see Fig. 2).

- At a certain distance $r$ from the root, skin and shear web widths are constants.

- The only action to be considered will be the out-ofplane bending momentum and the inertia tensor will be considered as a diagonal matrix (symmetrical cross-section from the point of view of stress distribution). The most exerted points will be assigned a distance from the flection axes equal to half the thickness.

On the other hand, there are two scenarios to be taken into account in the wind turbine blade design [13]:

- The first one analyzes the extreme loads that a wind turbine can suffer, which can be estimated using two simplified methods: parked under extreme winds and an operating gust condition
[14]. The first method calculates the extreme loads with the turbine in the parked condition in accordance with IEC and Germanisher Lloyd Class I design recommendations. In the second method the turbine is considered to be operating at constant speed during a $55 \mathrm{~m} / \mathrm{s}$ gust. Both load estimation approaches provide similar results.

- The second scenario refers to the analysis of the cycling loads in normal operation over the blade during the complete life of the wind turbine which diminishes the maximum strength the material is able to withstand.

A further knowledge of the methodology for blade design can be found in [12], but in this paper only a simplification of the second scenario has been taken into account.

The fatigue effect will be considered by assuming an amplitude variation of $18 \%$ around the mean rated value. Mean value is due to the out-of-plane flap-wise momentum and to the centrifugal forces. The momentum fluctuations are due to the wind shear, to the tower shadow, to gravitational forces, or, with lower frequencies, to weak wind gusts. Fatigue load spectra for different numbers of cycles will not be considered. It will be assumed a number of cycles equal to $2.1 \cdot 10^{8}$ cycles, obtained for the 20 -year life time of the blade, a rotational speed of $25 \mathrm{rpm}$, and an effective disposability of $80 \%$.

Instead of the amplitude variation it is more common to use the R-value to define the fatigue behaviour

$$
R=\frac{\sigma_{\min }}{\sigma_{\max }}=\left\{\begin{array}{c}
\frac{\sigma_{\text {mean }}-\sigma_{a}}{\sigma_{\text {mean }}+\sigma_{a}} \text { for tensile strengths } \\
\frac{\sigma_{\text {mean }}+\sigma_{a}}{\sigma_{\text {mean }}-\sigma_{a}} \text { for compressive strengths }
\end{array}\right.
$$

where $\sigma_{\min }$ and $\sigma_{\max }$ represent the minimum and maximum stress in a fatigue stress cycle (tension is considered positive and compression is negative) and $\sigma_{\text {mean }}$ and $\sigma_{a}$ are the mean value and the amplitude of the fatigue stress cycle. Assuming $\sigma_{a}=0.18 \sigma_{\text {mean }}$, the value for $R$ yields 0.7 for tensile strengths and 1.4 for compressive strengths (neglecting in a first stage the centrifugal forces).

Starting from the behaviour of a typical composite of glass reinforced epoxy, data for the maximum applied stress $\sigma_{u}$ can be obtained from [15]. This paper also proposes the following equation

$$
\sigma_{u}-\sigma_{\max }=a \sigma_{\max }\left(\frac{\sigma_{\max }}{\sigma_{u}}\right)^{b}\left(N^{c}-1\right)
$$

where $\sigma_{u}$ is the ultimate strength $(620 \mathrm{MPa}$ for tensile and $400 \mathrm{MPa}$ for compressive forces) and $a, b$ and $c$ are the fitting parameters, which depends on the value of $\mathrm{R}$. The input parameters are shown in Table I. 
Table I. Input data for the calculation of maximum strength $\sigma_{u}$

\begin{tabular}{|l|c|c|c|c|c|}
\hline Action & $R$ & $a$ & $b$ & $c$ & $\sigma_{u}$ \\
\hline Tensile & 0.7 & 0.04 & 2.5 & 0.45 & 620 \\
\hline Compressive & 1.4 & 0.06 & 3 & 0.15 & 400 \\
\hline
\end{tabular}

The value of $\sigma_{\max }$ yields $124 \mathrm{MPa}$ for the tensile strength and $290 \mathrm{MPa}$ for the compressive one. The more restrictive strength will be considered. The value for $\sigma_{\text {mean }}$ can be obtained next

$$
\sigma_{\text {mean }}=\frac{\sigma_{\max }}{1.18}=106 \mathrm{MPa}
$$

similar, although a bit lower, to $120-140 \mathrm{MPa}$ appearing in [12] or [15].

\section{B. Static analysis for the box-spar area}

This study starts from the following equation to calculate the span-wise strengths $\sigma_{r r}$ at a certain distance $r$ from the rotation axis at a point of the box-spar structure defined through its coordinates $y$ and $z$ ( $y$ and $z$ following the chord direction and its perpendicular line, respectively, in Fig. 6)

$$
\sigma_{r r}(r, y, z)=\frac{N_{r}(r)}{A(r)}+\frac{M_{z}(r)}{I_{z}(r)} y+\frac{M_{y}(r)}{I_{y}(r)} z
$$

where $N_{r}$ is the force in the direction of the blade due to centrifugal loads, $A(r)$ is the spar cap and shear web areas, $M_{z}(r)$ and $M_{y}(r)$ are the chord-wise and flap-wise momenta due to the resultant force component (see Fig. 5) and finally $I_{y}(r)$ and $I_{z}(r)$ are the momenta of inertia of the spar cap and shear web structure with respect to the chord line and to the axis perpendicular to it.

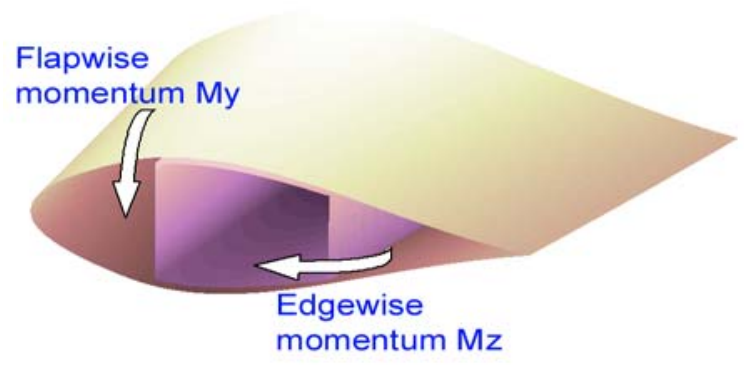

Fig. 5. Loads acting on a wind turbine blade

Figure 6 shows the higher value for the $\mathrm{z}$-axis component of the resultant force compared to the $y$-axis one, giving rise to significantly higher values for the flap-wise momentum $M_{y}$. In addition, $I_{y}(r)>>I_{z}(r)$. As a result, the second addend of (3) will be disregarded in the spar cap thickness analysis. In order to take into account the worst scenario, the value of $z$ in (3) corresponds to the most stressed point due to the flapwise momentum which is the farthest point from the median line. A value equal to half the thickness will be considered.

With regard to the structural reinforcement area, it is obtained by multiplying the section perimeter by the spar width. In fact, this width is not constant along the box-spar. In [14] the structural shear web was taken to be $5 / 3$ the thickness of the blade skins, and the spar cap reinforcement is $2 / 3$ of this outer skin. Thus, taking the thickness $\varepsilon^{\text {web }}$ of the structural shear web as the base, the area of the main spar can be expressed as the product of the parameter $k_{\text {perim }}^{\text {spar }}$, the chord $c h(r)$ and the structural shear web thickness.

$$
S^{\text {spar }}(r)=k_{\text {perim }}^{\text {spar }}(r) \cdot \operatorname{ch}(r) \cdot \varepsilon^{w e b}(r)
$$

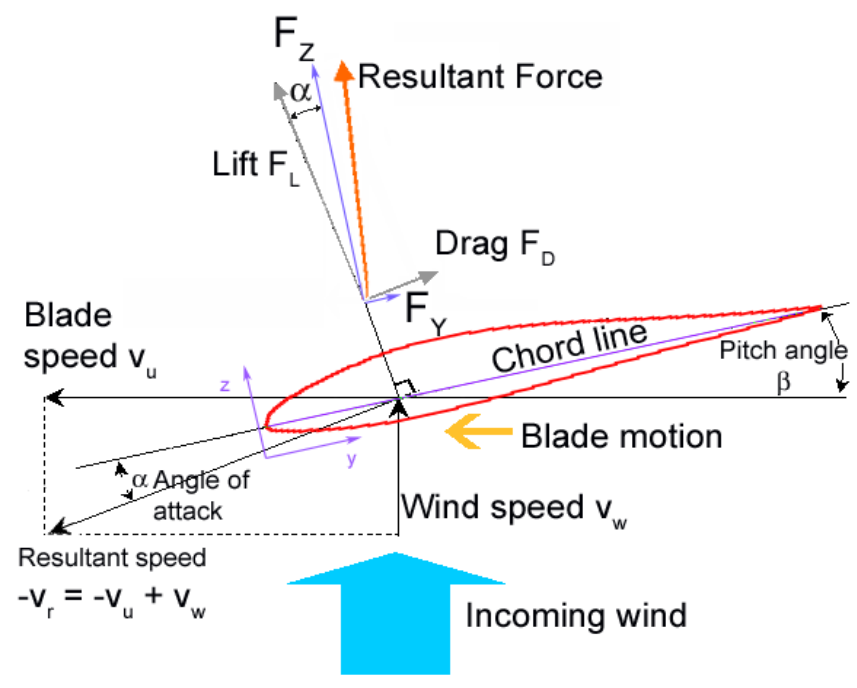

Fig. 6. Loads acting on a wind turbine blade

Analogously, a parameter $k_{l y}^{\text {spar }}$ can be defined such as

$$
\text { ¡Error! Vínculo no válido. }
$$

Hence the expression for the span-wise force yields

$$
N_{r}^{\text {web }}(r)=\rho_{G R P} \Omega^{2} \int_{r}^{L+R_{\text {hub }}} k_{\text {perim }}^{\text {spar }}(x) \operatorname{ch}(x) \varepsilon^{\text {web }}(x) d x
$$

where $\rho_{G R P}$ is the reinforced plastic density, $\Omega$ is the rotational speed in $\mathrm{rad} / \mathrm{s}, R_{H u b}$ is the hub radius, $L$ is the blade length , $\varepsilon^{\text {web }}(x)$ and $\operatorname{ch}(x)$ are the expressions for the shear web width and the blade chord as a function of the distance to the rotation axis.

Bending flap-wise momentum $M_{y}(r)$ can be calculated by integrating the force differentials shown in Fig. 6 whose expressions are

$$
\begin{aligned}
& d F_{L}(x)=\frac{\rho}{2} \operatorname{ch}(x) \cdot v(r)^{2} \cdot c_{l} \cdot d x \\
& d F_{D}(x)=\frac{\rho}{2} \operatorname{ch}(x) \cdot v(r)^{2} \cdot c_{d} \cdot d x
\end{aligned}
$$

where $\rho$ is the air density, $c_{l}$ and $c_{d}$ are the aerodynamical lift and drag coefficients, and $v_{r}(x)$ is the resultant relative velocity defined through its direction $\delta$ and its modulus $\left|v_{r}\right|$, which is determined by the rotational speed $\Omega$ through

$$
\begin{aligned}
& \delta=\tan ^{-1}\left(\frac{v_{\text {wind }}}{\Omega r}\right) \\
&\left|v_{r}\right|^{2} \cong \Omega^{2}\left(\frac{v_{\text {wind }}^{2}}{\Omega^{2}}+r^{2}\right)
\end{aligned}
$$


Thus, the flap-wise momentum $M_{y}(r)$ can be obtained through

$M_{y}(r)=\frac{\Omega^{2}}{2} \rho c_{l d} \int_{r}^{R_{h u b}+L} c h \cdot(x-r)\left(\frac{v_{\text {wind }}^{2}}{\Omega^{2}}+x^{2}\right) d x$

for $r>$ lim_aerod

$M_{y}(r)=\frac{\Omega^{2}}{2} \rho c_{l d} \int_{\text {lim_aerod }}^{R_{\text {hut }}+L} c h \cdot(x-r)\left(\frac{v_{\text {wind }}^{2}}{\Omega^{2}}+x^{2}\right) d x$

for $r \leq$ lim_aerod

where $c_{l d}=c_{l} \cos \alpha+c_{d} \sin \alpha, \alpha$ is the angle of attack and $\rho$ is the air density.

Introducing, for sake of clarity, the constant $K A_{\text {cent }}$ and the variable $K B(r)$, and after some calculations [1], the equations for the area $S(x)$ yields

$K A_{\text {cent }}=\frac{\rho_{G R P} \Omega^{2}}{\sigma_{\text {mean }}} \quad K B(r)=\frac{\Omega^{2} \rho \operatorname{th}(r) c_{l d} k_{\text {perim }}^{\text {spar }}}{4 k_{l y}^{\text {spar }}(r) \operatorname{ch}(r)^{2} \sigma}$

$S(r)=-e^{-\frac{\text { KAcent }}{2} r^{2}} \int_{r}^{R_{h u b}+L} e^{\frac{K A c e n t}{2} p^{2}}$

$\left(\frac{d K B(p)}{d p} \int_{\min (p, \text { lim_aerod })}^{R_{\text {hub }}+L}(x-p)\left(\frac{v_{\text {wind }}^{2}}{\Omega^{2}}+x^{2}\right) c h \cdot d x+\right.$

$\left.+K B(p) \int_{\min (p, \text { lim_aerod })}^{R_{\text {hub }}+L} c h\left(\frac{v_{\text {wind }}^{2}}{\Omega^{2}}+x^{2}\right) d x\right) d p$

Once derived the area $S(r)$, the web width $\varepsilon^{\text {web }}(r)$ can be obtained from (4). The shear web width obtained this way follows the distribution represented in Fig. 7. It can be seen that, due to the low value for the thickness at the tip, the width of the shear web is considerably larger than the width closer to the blade root.

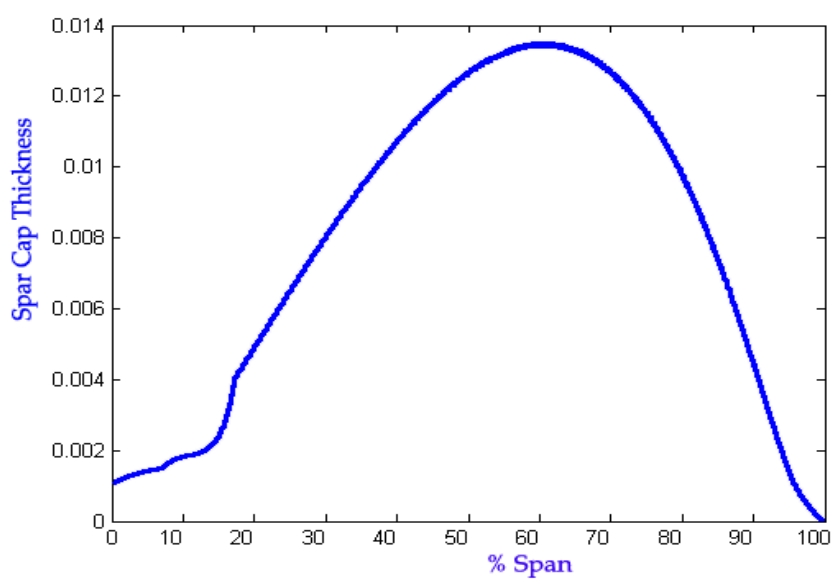

Fig. 7. Shear web width along the blade span.

However, difficulties arising during the manufacturing process make that a constant value for the shear web and the spar cap thickness is usually preferred. In order to provide for other materials which are also part of the blade (mainly the balsa core) the value previously obtained is multiplied by a factor which is greater near the root [16].

\section{C.Static analysis for the blade skin}

The same analysis can be made for the blade skin. This shell bears most of the edgewise bending loads which are due to the aerodynamical forces, but mainly to the weight force when the blade is in a horizontal position. It also bears the centrifugal forces as tensile ones. Instead of considering a calculated evolution of the skin width, a constant value for this value will be applied as well. In order to improve the reliability against extreme winds, a $50 \%$ increase of the spar thickness is also applied.

As mentioned before, [14] provides values for the approximated ratios between shear web skin and the outer skin, and also between shear web skin and the spar caps thickness. Taking into account these relationships, the total mass will depend on the shear web width. For a $1.9 \mathrm{~cm}$ skin $(35 \%$ over the maximum obtained in Fig. 7), a total mass of $3090 \mathrm{~kg}$ is obtained.

\section{Comparison of cumulative mass distributions}

The cumulated mass distribution along the span is shown in Fig. 8 in comparison to two typical cumulated mass distributions $[12,8]$.

It shows that making constant the value of the skins and reinforcing the cylindrical part of the blade, the cumulated mass distributions fit typical ones. Once the skin and spar cap areas along the span and the density of the glass reinforced plastic are known, the inertia constant $J$ and the centre of gravity from the blade root can be calculated

$J=4.34 \cdot 10^{5} \mathrm{~kg} \cdot \mathrm{m}^{2}$

$X c g=8.55 \mathrm{~m}$

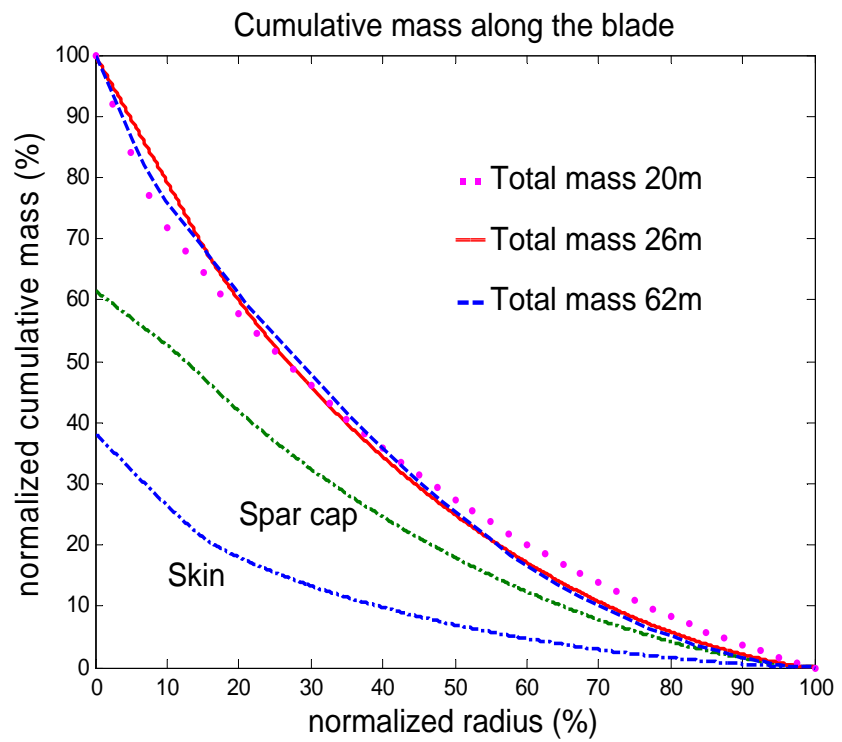

Fig. 8. Typical and calculated cumulated mass. 
The centre of gravity is similar to the one given by [17] or [18] for the same length blade.

\section{E Relationships weight blade-length blade.}

Applying the analysis deduced in subsection 4.B to different lengths, the values $\alpha=0,552$ and $\beta=2,645$ have been found for the relationship $M=\alpha \cdot L^{\beta}$. In Fig. 9, these values are compared to other ones obtained from the bibliography and catalogues. The sources for the other trends are given in [17] $\left(0.62 \cdot L^{2.63}\right)$, [19] $\left(1.50 \cdot L^{2.34}\right)$ and [20] $\left(1.6 \cdot L^{2.3}\right)$. The trend $\left(2.95 \cdot L^{2.13}\right)$ has been obtained by calculating the parameters of the curve that best fit the extracted data from different wind turbines [1].

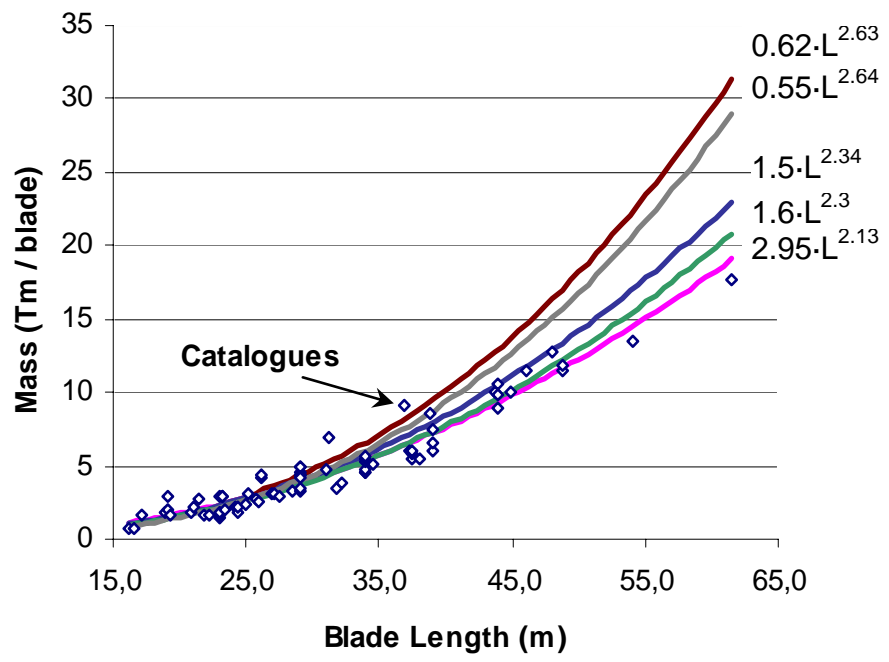

Fig. 9. Typical and calculated cumulated mass.

\section{Inertia time constant for a generic blade}

\section{A. Estimation of the inertia constant from the mass and length blade}

If the width of the spar-cap box and the skin do not vary along the blade, then an expression for the inertia constant $J$ of blades having geometry similar to the one analyzed can be obtained:

$$
J=k_{J} M L^{2}
$$

where $M$ and $L$ are the blade mass and length and $k_{J} \approx 0.212$.

The other distributions in Fig. 8 give rise to $k_{J}=0.222(20$ meter blade [12]) and $k_{J}=0.197$ (62 meter blade [8]).

If, on the other hand, the width of the spar-cap box and the skin would not be considered as a constant along the blade span, it can be proven by several numeric calculations that the value of $k_{J}$ keeps significantly constant in the range for blades with geometry similar to the one studied.

\section{B. Inertia time constant versus capacity}

The following expression can be used to relate inertia time constant, $H$, and the inertia constant, $J$ :

$$
H=z J \frac{\Omega_{g e n}{ }^{2}}{2 P n_{g b}{ }^{2}}=z J \frac{\left(\frac{f\left(1+s_{G}\right)}{n_{p p}} 2 \pi\right)^{2}}{2 P n_{g b}{ }^{2}}
$$

where $z$ is the number of blades, $\Omega_{\text {gen }}$ is the rotational speed of the generator, $f$ is the network frequency, $s_{G}$ is the rated generator slip (typically 0.01), $n_{p p}$ is the number of pole pairs, $P$ is the wind turbine capacity in watts, and $n_{g b}$ is the gearbox ratio.

In order to deduce the trend of the inertia time constant value along with the increasing capacity, the Power-toLength, the Weight-to-Length and the Gear_box_ratioto-Length relationships should be considered. The following approximate relationships have been correlated from technical wind turbine data [1]:

- Capacity as a function of the rotor diameter

$$
P[w] \cong k_{P} D^{\alpha p}=310 D^{2.01}
$$

- Mass of the blade as a function of its length

$$
M_{\text {Blade }} \cong k_{M} L^{\alpha_{M}}=2.95 L^{2.13}
$$

- Rotor diameter as a function of the blade length

$$
D \cong r e l_{D L} L=2.08 L
$$

- Gear box ratio as a function of the rotor diameter

$$
n_{g b} \cong k_{g b} D=1.186 D
$$

A representation of these data and their fitted relationship can be shown in Fig. 10-12.

Taking these approximate relations, the inertia time constant turns into

$$
H \cong k_{H} D^{\alpha_{H}}=2.63 \cdot D^{0.12}
$$

A similar expression can be estimated for the $H-P$ relationship (P expressed in watts):

$$
H \cong 1.87 \cdot P^{0.0597}
$$

\section{Other mechanical constants}

\section{A .Estimation of the remaining inertia time constant}

With regard to the hub inertia, this device weighs around one third of the rotor mass (without including shaft or gearbox), or analogously half the weight of the three blades. Assuming a maximum radius of 2 meters for a medium size wind turbine, the hub inertia can be estimated from (12), yielding a constant $H$ lower than $0.05 \mathrm{~s}$. The other components of the torque transmission system (gearbox, brake, fast shaft or slow shaft) do not have significant inertias either, and hence they will be omitted unless direct data are available.

By comparison, it is easier to find reliable values for the generator inertia. They show the great influence of the kind of generator on its inertia. For example, for a generator of about $1500 \mathrm{~kW}$, the inertia can vary from 


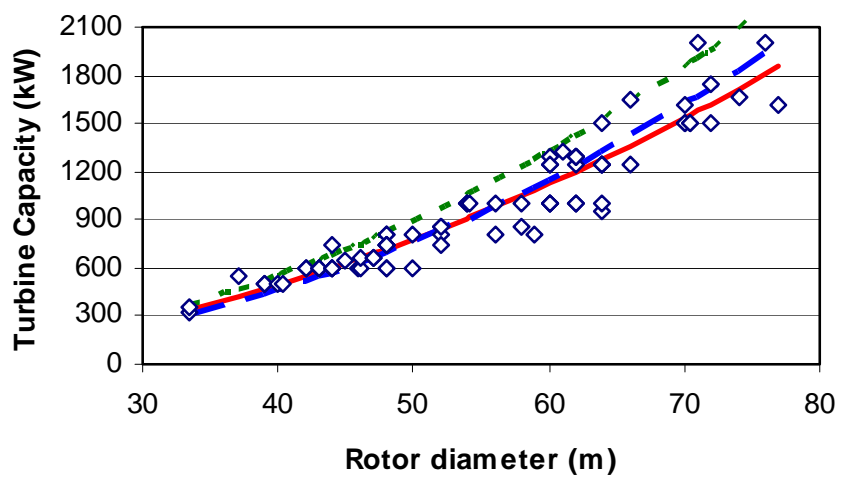

Fig. 10. Relationship Capacity-diameter.

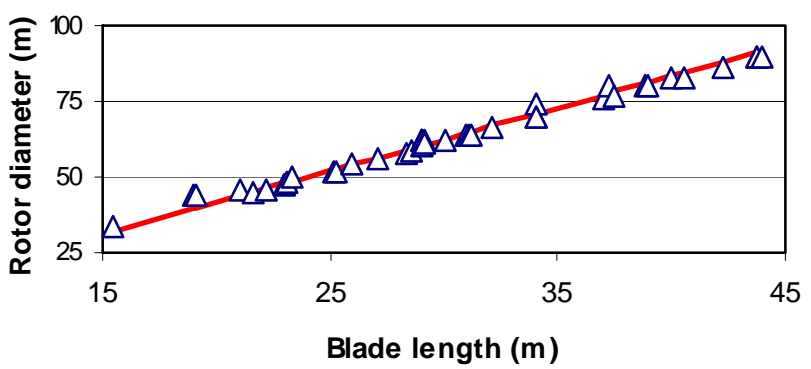

Fig. 11. Relationship Rotor diameter-Blade length.

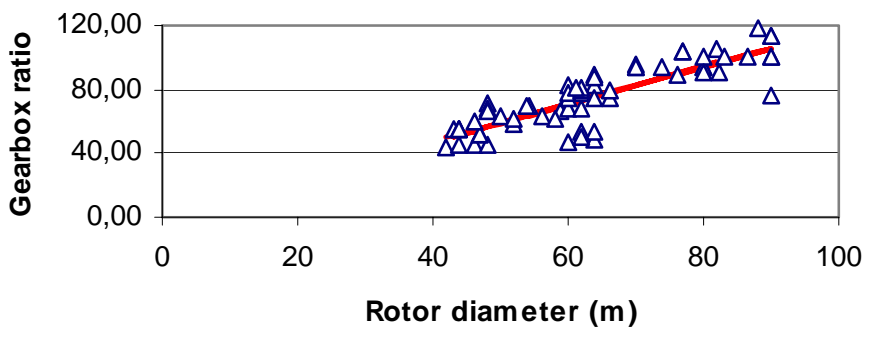

Fig. 12 Relationship Gear box ratio-Rotor Diameter. around $75 \mathrm{~kg} \mathrm{~m} \mathrm{~m}^{2}$ (generator Weier from Vestas V66$1.65 \mathrm{MW}$, rotor winding weight $1950 \mathrm{~kg}$, total weight 6473 $\mathrm{kg}$ ) for a wound rotor and around $35-50 \mathrm{~kg} \cdot \mathrm{m}^{2}$ for a squirrel cage generator. The generator inertia time constant $H$ in seconds results in a value of $0.63 \mathrm{~s}$ for the wound rotor and $0.29-0.45 \mathrm{~s}$ for the squirrel cage rotor. These values can be considered to be independent of the rated power

\section{B. Self damping}

This parameter, as well as the other mechanical transmission system parameters, does not have the same influence as the inertia. For fixed or narrow-range speed wind turbines, the effect of the self damping during the electrical connection to the grid is reduced to an almost constant antagonistic torque.

In order to estimate how self damping varies with the rated capacity, the resistant torque must be calculated from the aerodynamical drag force presented in (7).

The component of this force upon the rotation plane multiplied by the lever arm $r$ gives rise to the differential torque opposing the blade movement. Integration of these differential momenta for the three blades yields the total aerodynamical drag torque. Therefore, since the differential drag force varies with the diameter $\left(d F_{D}\right.$ varies with the chord and the chord varies with the diameter), and also does the lever arm and the upper integration limit, the drag torque varies with diameter cubed.

Assuming that the rotational speed $\Omega$ is inversely proportional to the diameter and the resistant torque due to the drag can be expressed in the simplest way as a friction torque (as done by PSCAD/EMTDC) through

$$
T_{\text {drag }}=S D\left[\frac{N \cdot m \cdot s}{\mathrm{rad}}\right] \cdot \Omega
$$

then self damping is expected to vary with $\mathrm{D}^{4}$.

Analogously as for the inertia, the resultant value can be transformed from the international system MKS to per unit p.u. through the expression

$$
S D[p . u .] \cong S D\left[\frac{N \cdot m \cdot d}{r a d}\right] \frac{\left(\frac{f}{n_{p p}} 2 \pi\right)^{2}}{P n_{g b}{ }^{2}}
$$

As indicated in (13) rated power varies practically as the square of the diameter, and the gear-box ratio varies as diameter does. This can suggest assuming that self damping in p.u. can be considered as a constant regardless of the turbine capacity.

¡Error! Vínculo no válido.

It was assumed that this value depended only on the drag. However it can be established that it also depends upon the friction and ventilation in the generator. These effects can be lumped in a single value.

Few references can be found with estimated or actual values for this parameter and the range for these values is very wide. For simulation purposes, a self damping close to $S D=0.05$ p.u. is frequently chosen $(0.052$ in [3] or 0.044 in [5]).

\section{Torsional stiffness and mutual damping}

This parameter connects the torque exerted by the drive force and the twist angle of the flexible coupling. For a flexible coupling in the slow shaft this proportionality yields (in MKS system)

$$
T[N \cdot m]=K^{l s}\left[\frac{N \cdot m}{m e c . r a d}\right] \cdot \theta[\text { mec.rad }]
$$

If all magnitudes are expressed in $p . u$, then

$$
K_{p u}^{l s}\left[\frac{p . u .}{e l . r a d}\right] \cong K^{l s} \frac{f \cdot 2 \pi}{P \cdot n_{g b}^{2} \cdot n_{p p}^{2}}
$$

and if the stiffness of the high speed shaft is also considered, then

$$
K_{p u}^{h s}\left[\frac{p . u .}{e l . r a d}\right] \cong K^{h s} \frac{f \cdot 2 \pi}{P \cdot n_{p p}^{2}}
$$

To consider the torsional stiffness for both slow and fast shaft, the equivalent constant results 


$$
K_{p u}^{e q}\left[\frac{p . u .}{\text { el. } r a d}\right]=\frac{K_{p u}^{l s}+K_{p u}^{h s}}{K_{p u}^{l s} \cdot K_{p u}^{h s}}
$$

A value of $K_{p u}=0.3$ p.u./el.rad can be used, which is similar to those indicated in $[4,6,9]$. Influence of this parameter on the system dynamics begins to be noticeable for lower values than aforementioned ones (very flexible couplings) and when the motor or resistant torque is suddenly lost, as in the case of a short-circuit with breaker opening.

With regard to the mutual damping $M D$ a similar expression should be used to translate the MKS value into p.u.

$$
M D[p . u] \cong M D\left[\frac{N \cdot m \cdot s}{r a d}\right] \frac{(f \cdot 2 \pi)^{2}}{P \cdot n_{p p}^{2} \cdot n_{g b}^{2}}
$$

and a value of about $M D=25$ is used in the scarce bibliography $[3,5]$ dealing with this parameter.

\section{Conclusions}

An analysis of the main mechanical parameters of a wind turbine rotor has been reported.

A static analysis has been made for a typical blade to obtain the theoretical distribution of the skin and the box-spar widths. On the other hand, a cuasi-constant distribution of these widths along the blade has also been analyzed that better fits actual distributions extracted from the bibliography.

An approximate but realistic expression to calculate the rotor inertia constant as a function of the blade mass and length has been deduced. Data from different manufacturers has been gathered in order to deduce expressions to estimate the inertia time constant as function of the rotor diameter and the turbine capacity.

It has also been proven that the self damping (in p.u.) is not significantly influenced by the wind turbine capacity.

\section{References}

[1] A.G. González Rodriguez, "Improvement of a Fixed-Speed Wind Turbine Soft-Starter Based on a Sliding-Mode Controller", $\mathrm{PhD}$ Thesis. Department of Electrical Engineering, University of Seville, 2006.

[2] Z. Saad-Saoud and N. Jenkins, "A modular approach to simulating wind farm dynamics", 12th Power System Computation Conference, Dresden, pp 1235-1241, August 19-23 1996.

[3] S.A. Papathanassiou and M.P. Papadopoulos, "Dynamic behaviour of variable speed wind turbines under stochastic wind". IEEE Transactions on EnergyConversion, Vol. 14(No. 4):pp. 1617-1623, December 1999.
[4] T. Petru, "Modeling of wind turbines for power system studies", Technical report no 3911, Chalmers University of Technology, GÄoteborg, Sweden, 2001.

[5] P. Ledesma, J.J. Vicente and J. Usaola, "Análisis dinámico de un parque eólico de turbinas de velocidad fija", 6as Jornadas Luso-Espanholas de Engenharia Electrotécnica, Lisboa, julio 1999, pp. 37-44.

[6] V. Akhmatov, H. Knudsen and A.H. Nielsen, "Modelling and transient stability of large wind farms", Electrical Power and Energy Systems, Elsevier Science Ltd, February 2002.

[7] E.S. Abdin and W. Xu, "Control design and dynamic performance analysis of a wind turbine-induction generator unit", IEEE Transactions on Energy Conversion, Vol. 15, No. 1, March 2000, pp. 91-96.

[8] H.J.T. Kooijman, C. Lindenburg, D. Winkelaar, and E.L. Van der Hooft, "Aero-elastic modelling of the Dowec $6 \mathrm{MW}$ pre-design in Phatas", September 2003.

[9] Johannes Gerlof Slootweg. "Wind Power. Modelling and Impact on Power System Dynamics. PhD thesis", December 2003.

[10] Aleksandar Radovan Katancevic. "Transient and Dynamic Stability on Wind Farms". PhD thesis, March 2003. Helsinki University of Technology.

[11] Janaka B. Ekanayake, Lee Holdsworth, XueGuang Wu, and Nicholas Jenkins, "Dynamic modeling of doubly fed induction generator wind turbines", IEEE Transactions on Power Systems, 18(No. 2), pp. 803-809, May 2003.

[12] Tony Burton, David Sharpe, Nick Jenkins and Ervin Bossanyi. Wind energy handbook, John Wiley \& Sons, Ltd, 2001

[13] CEI Commission Electrotechnique Internationale. "International standard 61400-1, wind turbine generator systems, Part 1 Safety requirements". Technical report, 1999.

[14] Sandia National Laboratories. "Parametric study for large wind turbine blades" Technical report, TPI Composites, Inc., August 2002.

[15] J.F. Mandell, D.D. Samborsky, N.K. Wahl, and H.J. Sutherland. "Testing and analysis of low cost composite materials under spectrum loading and high cycle fatigue conditions". ICCM14, SME/ASC, 2003. paper \# 1811

[16] K.J. Jackson, M.D. Zuteck, C.P. van Dam, K.J. Standish, and D. Berry. "Innovative design approaches for large wind turbine blades", in Wind Energy, volume 8, pp. 141-171. John Wiley \& Sons, 2005.

[17] J.L. Rodríguez Amenedo, J.C. Burgos Díaz, and S. Arnalte Gómez, "Sistemas Eólicos de Producción de Energía Eléctrica". Editorial Rueda S.L., 2003.

[18] C. Kong, J. Bang, and Y. Sugiyama, "Structural investigation of composite wind turbine blade considering various load cases and fatigue life", in Elsevier, Energy, Science Direct. 2004.

[19] Offshore technology, http://www.offshorewindenergy. org/caowee/indexpages/Offshore technology.php,

November 2004

[20] Dayton Griffin, "Evaluation of design concepts for adaptive wind turbine blades", Technical report, Sandia National Laboratories, Albuquerque, August 2002. 